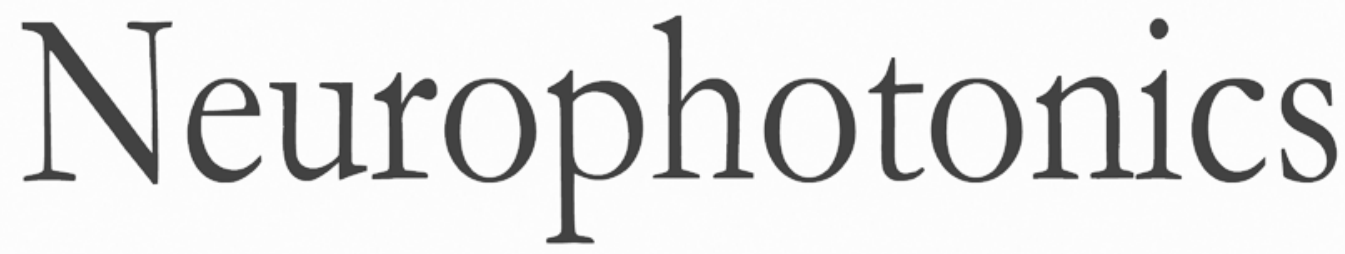

\title{
Characterization of the relative contributions from systemic physiological noise to whole-brain resting-state functional near-infrared spectroscopy data using single- channel independent component analysis
}

Ardalan Aarabi
Theodore J. Huppert 


\title{
Characterization of the relative contributions from systemic physiological noise to whole-brain resting-state functional near-infrared spectroscopy data using single-channel independent component analysis
}

\author{
Ardalan Aarabi ${ }^{\mathrm{a}, \mathrm{b}}$ and Theodore J. Huppert ${ }^{\mathrm{c}, \mathrm{d}, *}$ \\ aUniversity of Picardie Jules Verne, Faculty of Medicine, Amiens 80036, France \\ bUniversity Research Center (CURS), University Hospital, GRAMFC-Inserm U1105, Amiens 80054, France \\ 'University of Pittsburgh, Department of Radiology, 4200 Fifth Avenue, Pittsburgh, Pennsylvania 15260, United States \\ dUniversity of Pittsburgh, Department of Bioengineering, 4200 Fifth Avenue, Pittsburgh, Pennsylvania 15260, United States
}

\begin{abstract}
Functional near-infrared spectroscopy (fNIRS) is a noninvasive neuroimaging technique used to measure changes in oxygenated hemoglobin (oxy-Hb) and deoxygenated hemoglobin (deoxy-Hb) in the brain. In this study, we present a decomposition approach based on single-channel independent component analysis (scICA) to investigate the contribution of physiological noise to fNIRS signals during rest. Single-channel ICA is an underdetermined decomposition method, which separates a single time series into components containing nonredundant spectral information. Using scICA, fNIRS signals from a total of 17 subjects were decomposed into the constituent physiological components. The percentage contribution of the classes of physiology to the fNIRS signals including low-frequency (LF) fluctuations, respiration, and cardiac oscillations was estimated using spectral domain classification methods. Our results show that LF oscillations accounted for $40 \%$ to $55 \%$ of total power of both the oxy- $\mathrm{Hb}$ and deoxy $-\mathrm{Hb}$ signals. Respiration and its harmonics accounted for $10 \%$ to $30 \%$ of the power, and cardiac pulsations and cardio-respiratory components accounted for $10 \%$ to $30 \%$. We describe this scICA method for decomposing fNIRS signals, which unlike other approaches to spatial covariance reduction is applicable to both single- or multiple-channel fNIRS signals and discuss how this approach allows functionally distinct sources of noise with disjoint spectral support to be separated from obscuring systemic physiology. ๑ 2016 Society of Photo-Optical Instrumentation Engineers (SPIE) [DOI: 10.1117/1.NPh.3.2.025004]
\end{abstract}

Keywords: near-infrared spectroscopy; oxygenated/deoxygenated hemoglobin; physiological noise; single-channel independent component analysis; signal decomposition; resting state.

Paper 16006R received Jan. 18, 2016; accepted for publication May 10, 2016; published online Jun. 6, 2016.

\section{Introduction}

Functional near-infrared spectroscopy (fNIRS) is a noninvasive optical imaging technique that provides a measure of changes in regional oxygenated hemoglobin (oxy- $\mathrm{Hb}$ ) and deoxygenated hemoglobin (deoxy-Hb) concentrations in the cortex of the brain with a high sample rate. ${ }^{1,2}$ Similar to functional magnetic resonance imaging methods, this technique is sensitive to the hemodynamic changes in the brain due to blood flow and oxygen saturation changes. While these vascular signals are temporally slow compared to electrophysiological changes, fNIRS instruments often oversample at acquisition rates of tens to hundreds of samples per second, which is fast enough to avoid aliasing of most physiological sources of noise in the vascular signal. More specifically, fNIRS records changes in the absorption of light in the red to near-infrared range $(650$ to $900 \mathrm{~nm})$ in which oxy-Hb and deoxy-Hb have distinct absorption spectra. By knowing the molar extinction coefficients of oxy-Hb and deoxy-Hb at specific wavelengths, their temporal concentration changes can be calculated using the modified Beer-Lambert

*Address all correspondence to: Theodore J. Huppert, E-mail: huppertt@ upmc .edu law. ${ }^{3}$ Cerebral hemodynamic changes related to underlying neuronal brain activity are recorded using fNIRS sensors placed on the surface of the scalp. Previous fNIRS studies have been used to study stimulus-induced cerebral activation during visual, motor, and cognitive tasks (see Ferrari and Quaresima ${ }^{4}$ and Boas et al. ${ }^{5}$ for reviews). fNIRS has also been used to characterize spontaneous resting brain activity. ${ }^{6-8}$

A potential limitation of fNIRS is contamination from unwanted physiological noise related to autonomic blood pressure, respiratory signals, and cardiac pulsation. ${ }^{9}$ The sensitivity of fNIRS measurements falls off exponentially with depth to reach a penetration of $\sim 5$ to $10 \mathrm{~mm}$ into the cortex of the brain. This results in a high oversensitivity to superficial physiological noise in the scalp, especially due to the skin flow, which has been shown to have about a $16 \%$ contribution to fNIRS changes. ${ }^{10}$ Previous work by Franceschini et al. ${ }^{11}$ showed that the spatio-temporal distribution of these physiological signals varies with underlying arterial and venous vascular densities between anterior and posterior regions of the head. They observed a specific spatial propagation pattern for blood 
pressure signals and heart rate activities moving periodically from the anterior to the posterior and then middle regions of the brain in normal subjects.

Contamination by systemic physiology poses several challenges to the analysis and interpretation of fNIRS neuroimaging data. The functional connectivity within different cerebral regions can be highly affected by physiological noise. ${ }^{12}$ In the presence of systemic physiology, it is difficult to determine the portion of the fNIRS signals attributed to oscillations arising from neural connectivity in the brain. Within a limited range, some low- and high-frequency noise components may be removed using simple bandpass filtering (reviewed in Huppert et al. ${ }^{9}$ ). For example, to suppress high-frequency instrument noise and the fast cardiac oscillations, low-pass filtering methods such as moving averaging and Wiener filtering have been used efficiently. ${ }^{13}$ Similarly, high-pass filtering methods are used to eliminate low-frequency (LF) drift as well as LF activities such as blood pressure oscillations in the range 0.08 to $0.12 \mathrm{~Hz}^{9}{ }^{9}$ However, special care must be taken in handling signals with overlapping frequency bands, as it is the case between blood pressure and cerebral hemodynamic response, and between blood pressure, respiratory signals, and cerebral hemodynamic response. ${ }^{9}$ Similarly, due to the transient nature of motion artifacts, more sophisticated algorithms have been developed such as methods based on wavelet filtering, ${ }^{14}$ Wiener filtering, ${ }^{13}$ or independent component analysis (ICA). ${ }^{15}$ External physiological recording such as respiratory signals recorded using a chest belt, blood pressure, and cardiac signals have also been used in standard regression methods. ${ }^{9,16}$ The assumption in regression analysis is that there is a timeinvariant linear relationship between independently measured physiological signals and cerebral hemodynamic changes measured by fNIRS. ${ }^{17}$ Diamond et al. ${ }^{16}$ demonstrated that the relationship between fNIRS signals and external physiological measurements could be nonstationary in time, reflecting a complex and probably nonlinear relationship between systemic and cerebral physiology. More recently, dedicated fNIRS measurements from short distance $(<1 \mathrm{~cm})$ light source-detector spacing, which only penetrate the scalp layer, can be used to provide a more regional estimate of systemic physiology, which is presumably less sensitive to these nonlinear and nonstationary effects. ${ }^{18}$ Finally, the spatial structure of physiological noise can also be exploited to remove systemic physiological contaminations. Spatial covariance reduction methods based on principal component analysis (PCA) remove spatial eigenvectors related to physiological noise with global spatial effects, such as heart rate and blood pressure. ${ }^{11}$ Spatially unconstrained, decomposition methods based on ICA separate fNIRS signals into additive components such as brain hemodynamic responses, signals reflecting baseline physiological changes, and other signals related to respiration, vasomotion, noise, and movement artifacts. ${ }^{19}$

The characterization of slow resting-state oscillations in brain activity for connectivity analysis poses additional challenges in fNIRS due to the contamination of additional LF vascular changes with no direct coupling to neuronal activities. ${ }^{20}$ These vascular changes are regional and may be generated by the auto-regulation of regional cerebral blood flow. ${ }^{21}$ However, some regional cerebral hemodynamic changes are strongly associated with neurovascular coupling partially regulated by the autonomic control. ${ }^{22}$ There are other factors such as thermoregulation and metabolic demand that contribute to local cerebral hemodynamic changes, ${ }^{23}$ which are directly reflected in fNIRS measurements. Therefore, any tools to characterize physiological noise should take into account local changes in systemic physiological fluctuations. The characterization of fNIRS signal including both neural and vascular components becomes more difficult during the resting state. This is basically because fNIRS measurements include spontaneous resting-state neural activity as well as systemic and local physiological fluctuations with amplitudes comparable with that of task-induced brain hemodynamic activities. ${ }^{24}$

This paper presents a single-channel decomposition method based on the single-channel ICA (scICA) $)^{25}$ to characterize the physiological contributions of oxy- $\mathrm{Hb}$, deoxy- $\mathrm{Hb}$, and total hemoglobin (total-Hb) recordings from fNIRS. The scICA method, as a subspace approach, was used to separate these time series into their underlying sources, which were then used to estimate the contribution of the constituent components including LF oscillations, respiration, and cardiac pulsation to the original fNIRS signals. We applied the scICA method to the fNIRS data from a previously published dataset of 17 human subjects at rest, as described in Franceschini et al. ${ }^{11}$ The contribution of various sources of physiological noise was then estimated for different cerebral regions across the head. We evaluated the results at single-subject and group levels.

\section{Methods}

\subsection{Subjects and Experimental Setup}

The optical data used in this study were obtained from the experimental data previously reported in Franceschini et al. ${ }^{11}$ The resting fNIRS data have been collected from 17 healthy adults (age range $35 \pm 12$ years) lying motionless in a supine position in a quiet dark room. All subjects underwent two to three resting-state sessions of fNIRS measurements, each $300 \mathrm{~s}$. The fNIRS data have been collected with a continuous-wave optical system (CW5 Imager, TechEn Inc., Milford, Massachusetts) comprising 32 laser diodes (average output power of $\sim 1 \mathrm{~mW}$ ) at 690 and $830 \mathrm{~nm}$ and 16 avalanche photodiodes. ${ }^{11}$

A whole-head probe has been designed to cover the frontal, parietal, temporal, and occipital lobes of the brain for each subject. Figure 1 shows the schematic of the emitter/detector configuration on the head and the channels defined as the midpoints between pairs of optodes. In total, 50 channels have been used for data collection with a minimum interoptode distance of $3 \mathrm{~cm}$ (see Franceschini et al. ${ }^{11}$ for more details). As shown in Fig. 1, the fNIRS channels were grouped into eight cerebral regions (left/right frontal, temporal, parietal, and occipital regions) for single-subject and group analyses. The optical data were converted to optical density changes as described in Huppert et al. ${ }^{9}$ The modified Beer-Lambert law was used to calculate the concentration change of deoxy-Hb and oxy-Hb based on the differences in the absorption for the two wavelengths. The concentration in total-Hb was calculated by summing the concentrations in deoxy-Hb and oxy-Hb. The average differential path-length factor was assumed to be 6 for both wavelengths. ${ }^{26}$

During fNIRS data recordings, concurrent independent measurements of cardiac activity, respiration, and blood pressure has been made to assess their relationship to spontaneous cerebral hemodynamic fluctuations for each subject. For this purpose, a finger pulse oximeter (Nonin model 8600) has been used to record continuously the arterial saturation and the heart rate from 


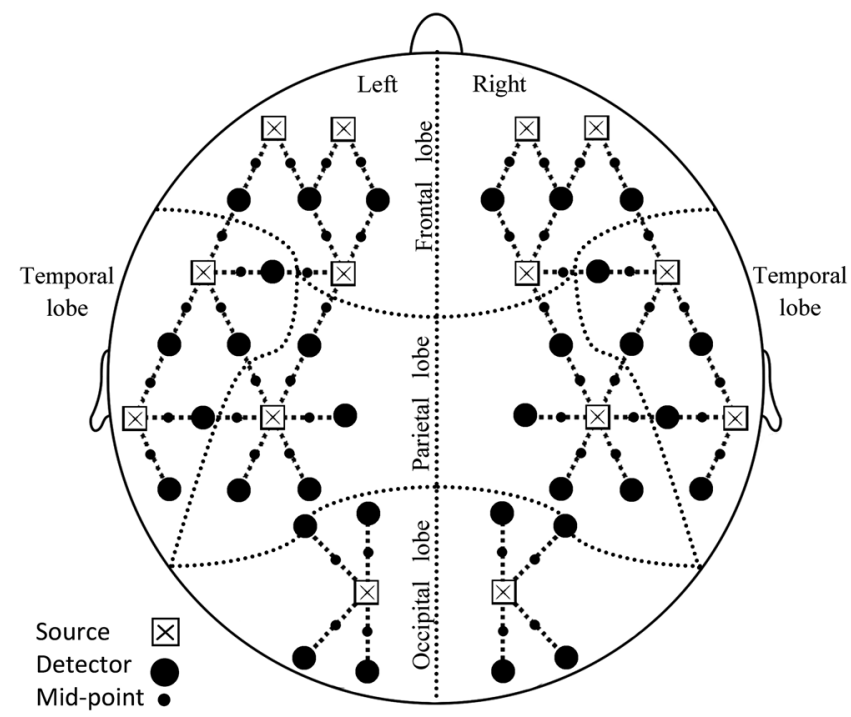

Fig. 1 Probe geometry used for data collection.

each subject's left hand. The arterial blood pressure (ABP) has also been measured using a custom-built beat-to-beat noninvasive blood pressure sensor. ${ }^{11}$ Finally, the respiratory signal has been measured by using a strain gauge respiratory belt (Sleepmate/Newlife Technologies, Resp-EZ). The external systemic physiology signals have been recorded synchronously with the optical data using a National Instrument 6023E card with a sampling rate of $25 \mathrm{kHz}$. The acquired external data were down sampled to match the sampling rate of the optical measurements $(10 \mathrm{~Hz})$.

\subsection{Single-Channel Decomposition of Functional Near-Infrared Spectroscopy Signals}

Figure 2 shows the decomposition process comprising three main stages: preprocessing, predecomposition, and single-channel decomposition. In the preprocessing step, the fNIRS signals were first bandpass filtered. Noisy channels were then rejected. Finally, motion artifacts were removed using the spatial ICA. The clean fNIRS signals of all channels were decomposed using the scICA method. We developed a procedure to estimate the optimal number of independent components (sources) for each channel based on the identification of the frequency bands associated with LF oscillations, respiration, and cardiac activity as well as those related to cardio-respiratory interactions. Finally, independent components associated with each frequency band were identified and back-projected onto the measurement space to recover the multichannel fNIRS signals corresponding to that frequency band. These steps will be described in more detail in the following sections. All data processing was done in MATLAB R2011b (The Mathworks, Natick, Massachusetts).

\subsubsection{Preprocessing}

Bandpass filtering. The fNIRS data including the deoxy$\mathrm{Hb}$, oxy- $\mathrm{Hb}$, and total-Hb signals were bandpass filtered using a fourth-order Butterworth filter to remove LF drift (below $0.01 \mathrm{~Hz}$ ), high-frequency instrument noise, and fast oscillations (above $1.5 \mathrm{~Hz}$ ).

Noisy channel removal. We discarded emitter-detector pairs (channels) with low signal-to-noise ratio (SNR) resulting from poor contact with the scalp. We used two complementary approaches to identify bad channels with poor-quality signals. Since bad channels tended to have higher variances, we first identified channels with variances greater than the average variance computed over all channels in the time domain. Then, the channels with high variance were tested for the presence of cardiac and other fluctuations considered as a sign of the quality of optical data. ${ }^{9}$ For this purpose, we identified visually the frequency bands of LF oscillations, respiratory, and cardiac signals by analyzing the power spectral density (PSD) of each subject's external systemic physiology signals within the frequency ranges reported in Ref. 27. Then, the relative spectral powers of these frequency bands were computed for the high-variance channels. The relative power of each frequency band of interest was defined as the ratio of the spectral power in that frequency band to the total power. Finally, the high-variance channels showing relative power $<25 \%$ within all three frequency bands were discarded from further analysis. This threshold was chosen based on the recommendations in Mesquita et al. ${ }^{8}$ to discard fNIRS channels with very poor coupling to the head, which have little to no physiological components to the signal.

Motion artifact reduction. For each subject, to further increase the quality of the deoxy- $\mathrm{Hb}$, oxy- $\mathrm{Hb}$, and total-Hb data, we removed motion artifacts from the multichannel fNIRS data using spatial-ICA. ${ }^{28,29}$ In the spatial-ICA approach, a data matrix $X$ of $N$ (observations, herein fNIRS data) rows by $P$ (measurement sites, herein fNIRS channels) columns is assumed to be linearly mixed of non-Gaussian signals generated from $M(\leq P)$ unknown statistically independent sources $(S)$

$X=A \cdot S$.

The main purpose of the decomposition using ICA is to find an "unmixing matrix" $W$ (the inverse of the mixing matrix $A$ ). $W$ is then used to separate independent sources as

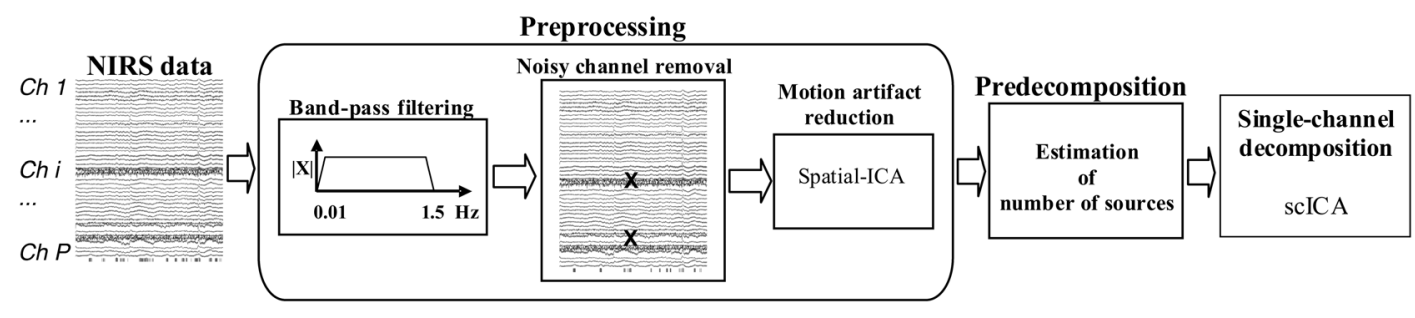

Fig. 2 Schematic of the single-channel decomposition process. 
$\hat{S}=W \cdot X$

Among the methods suggested in the literature, ${ }^{28}$ we used the deflationary kurtosis-based fastICA algorithm, which has been widely used to estimate $A$ and $W$ based on maximization of nonGaussianity, considered as a measure of statistical independence. In this approach, the kurtosis, defined as the normalized fourth-order marginal cumulant, ${ }^{30}$ is used as a contrast function to measure the non-Gaussianity

$k(W)=\frac{E\left\{|S|^{4}\right\}-2 E^{2}\left\{|S|^{2}\right\}-\left|E\left\{S^{2}\right\}\right|^{2}}{E^{2}\left\{|S|^{2}\right\}}$,

where $S$ refers to components and $E\{\cdot\}$ denotes the expectation. The first step in the fastICA algorithm is centering the observed vector $X$ and whitening it using PCA. Then, the deflationary kurtosis-based fastICA algorithm searches for a $W$, such that the projection $(W \cdot X)$ maximizes the contrast function [Eq. (3)] using a gradient-based update with a fixed step size. ${ }^{30}$

In general, fastICA is sensitive to initial values of $W .^{30}$ To tackle this problem, we used a modified version of fastICA, called robustICA, which employs an exact line search of the normalized kurtosis to find global maxima along given search directions. This approach has shown to be computationally fast, efficient, and insensitive to initial conditions. ${ }^{30}$ More importantly, robustICA estimates the optimal step size, which enhances the robustness of the algorithm in the presence of saddle points and local extrema in the contrast function.

To perform robustICA, the first step was to prewhiten the fNIRS data using the standard PCA approach by retaining $99 \%$ of the data variance. Then, the data were decomposed into independent components by robustICA. To identify channels affected by motion artifacts, we used the kurtosis-based method, ${ }^{31}$ in which kurtosis has been defined as a measure of the "peakedness" of the probability distribution. We removed the components having probability distribution sharply peaked at the center (a kurtosis value $>25$ ) and then back-projected the multichannel fNIRS data using the remaining components. A kurtosis value of 25 as the threshold is close to the threshold used in Chiarelli et al., ${ }^{32}$ which described a similar kurtosisbased selection criteria for wavelet filtering of fNIRS data. Normally distributed noise will have a kurtosis close to a value of 3 .

\subsubsection{Single-channel decomposition}

In this stage, the multichannel fNIRS data (deoxy-Hb, oxy-Hb, or total-Hb) were decomposed into their underlying components (sources) using the scICA method. The main assumption of the scICA method is that the observed signals are linear mixtures of a number of statistically independent source signals with disjoint spectral support. ${ }^{33}$

In this method, the fNIRS signal of any given channel $p$ was first mapped into a multidimensional space using the time delay embedding method as follows: ${ }^{33}$

$$
X_{j}=\left[\begin{array}{cccc}
x_{t}^{j} & x_{t+\tau}^{j} & \cdots & x_{t+N \tau}^{j} \\
x_{t+\tau}^{j} & x_{t+2 \tau}^{j} & \cdots & x_{t+(N+1) \tau}^{j} \\
\vdots & \vdots & \ddots & \vdots \\
x_{t+(m-1) \tau}^{j} & x_{t+m \tau}^{j} & \cdots & x_{t+(m-1+N) \tau}^{j}
\end{array}\right],
$$

where $x_{t}^{j}$ is the sample point of channel $j$ at time $t, \tau$ is the lag term, and $m$ is the embedding dimension (or number of lags). In this representation, the dynamics of the brain hemodynamic system generating the measured fNIRS data was constructed by creating a series of delay vectors from the original signal. ${ }^{33}$ In MATLAB, this can be constructed using the function "convmtx" of the data vector $x$ of size $<1$ by $N>$ with $m * \tau$ lags and then keeping only every $\tau$ th row. The matrix $X_{j}$ $(m$-by- $N)$, therefore, contains shifted versions of the original data and was constructed for all fNIRS channels $p, p=1: P$. Since, in this study, the fNIRS data have been optimally sampled ( $f_{s} \sim 10 \mathrm{~Hz}$ considered to be fast enough to capture hemodynamic changes related to the cardiac activity) and the lowest frequency of interest was $0.01 \mathrm{~Hz}$, we set $\tau$ and $m$ to 1 and 1000 , respectively (as recommended in Jimenez-Gonzalez and James $^{33}$ ). Each constructed $X$ was prewhitened using the singular value decomposition method ${ }^{34}$ and decomposed by robustICA into its spectrally independent components.

Our main problem was to determine the optimal number of ICs (sources). In the standard ICA applied to multichannel electroencephalography data, the number of ICs is usually restricted to the number of sensors. ${ }^{35}$ Concerning the singlechannel ICA, by definition, the maximum number of components for the decomposition of any channel's fNIRS signal could not exceed $m$, the number of delayed vectors in the matrix $X_{j}$, which was previously constructed for each channel. However, when a large number of components is used for decomposition, it is difficult to determine the physiological relevance of the components having spurious spectral peaks. We developed an estimation procedure comprising two stages to determine the optimal number of ICs associated with physiological sources for each subject's multichannel fNIRS data on a channel-by-channel basis (see Sec. 2.2.3 for details). The estimation procedure was repeated for all $P(\leq 50)$ channels and 17 subjects.

\subsubsection{Estimation of optimal number of sources}

For each subject and channel, the estimation procedure was employed in two steps to determine the optimal number of ICs associated with physiological sources.

Step 1: In the first stage of the estimation procedure, for each subject's multichannel fNIRS data (deoxy-Hb, oxy-Hb, or total-Hb), we created a frequency-band matrix $M_{\mathrm{FB}}$ with $N_{\mathrm{fb}}$ rows, where $N_{\mathrm{fb}}$ is the number of frequency bands of interest, and two columns including lower- and upper-frequency limits.

First, an average normalized PSD was computed for each fNIRS channel by decomposing its signal into $K$ independent components using the single-channel decomposition method described in Sec. 2.2.2, where $K$ was the number of the retained principal components (PCs) that accounted for $99 \%$ of the variance of the channel's signal. Then, the PSDs of the $K$ independent components were computed using the Welch spectral estimation method ${ }^{36}$ with a hamming window of length 1000 samples and an overlap of $20 \%$. The window lengths and overlaps were chosen empirically to achieve high-frequency resolution $(0.01 \mathrm{~Hz})$ and good spectral smoothness for the fNIRS data length of 3000 samples (300 s). Finally, the PSDs of the $K$ independent components were normalized to their total power, and then averaged to obtain the average normalized PSD $\left(\mathrm{PSD}_{\mathrm{AN}}\right)$ for the channel. 
It can be noted that the normalization procedure was necessary to avoid spectral peaks of different magnitudes masking each other during averaging. The whole procedure was repeated for all $P(\leq 50)$ channels. Then, a spatially averaged $\mathrm{PSD}\left(\mathrm{PSD}_{\mathrm{SA}}\right)$ was computed by averaging the $\mathrm{PSD}_{\mathrm{AN}}$ of all $P$ channels. By averaging across all channels, we removed spurious spectral peaks of single channels. On the other hand, global spectral peaks with low power were retained through the normalization process. All frequency bands of the $\mathrm{PSD}_{\mathrm{SA}}$ were identified by finding the points of inflection using the first and second derivatives. In $\mathrm{PSD}_{\mathrm{SA}}$, the spectral peaks (local maxima) were identified by finding the zeros of the first derivative of the spectrum. We then used the zeros of the second derivative of the spectrum to identify the upper and lower limits of each frequency band, i.e., the inflection points on the spectrum, at which the concavity changes from up to down or vice versa. The $N_{\mathrm{fb}}$ spectral peaks and frequency bands were stored in the matrix $M_{\mathrm{FB}}$, which was then used to estimate the number of sources for each channel in the multichannel fNIRS data.

Step 2: In the second stage of the estimation procedure, for each channel in the fNIRS recording, the optimal number of sources (IC components) was determined between the minimum $\left(\mathrm{NS}_{\min }\right)$ and maximum $\left(\mathrm{NS}_{\max }\right)$ number of sources. $\mathrm{NS}_{\min }$ was set to 3 because in this study, we mainly dealt with three main physiological frequency bands associated with (1) LF fluctuations caused by hemodynamic changes in ABP and local brain hemodynamic activity; (2) respiration (with a spectral peak at $f_{R}$ ); and (3) cardiac activity (with a spectral peak at $\left.f_{C}\right)$. $\mathrm{NS}_{\max }$ was determined using the prewhitening procedure by finding the minimum number of PCs $\left(N_{P C}\right)$ retaining $99 \%$ of the fNIRS data variance. Therefore, the optimal number of sources was estimated in the interval $\left[3 N_{P C}\right]$.

For each fNIRS channel, the estimation procedure started by first decomposing the channel fNIRS signal into three components $\left(\mathrm{NS}_{\min }=3\right)$. Then, for each component, a normalized PSD ( $\mathrm{PSD}_{\mathrm{AN}}$ ) was calculated and analyzed to identify its frequency bands with the method described earlier in this section. The components were grouped based on their frequency content. For this purpose, the number of groups was set to $N_{\mathrm{fb}}$, i.e., the number of frequency bands included in the matrix $M_{\mathrm{FB}}$ of the channel. Therefore, for each frequency band in $M_{\mathrm{FB}}$, all the components whose PSD $_{\mathrm{AN}}$ showed a spectral peak within that frequency band were grouped. Each component was included in multiple groups if its PSD $_{\mathrm{AN}}$ exhibited spectral peaks in all the frequency bands associated with the groups. Then, the grouped ICs were projected back to the original fNIRS signal space and the resulting signal was filtered within the frequency band of the class to remove out-of-band spectral peaks.

At each run, the residual of the decomposition procedure using $N_{S}$ sources was computed by subtracting the sum of the reconstructed signals from the original signal. The relative power of the residual was calculated as the ratio of the power of the residual and the total power of the original signal.

After each run, $N_{S}$ was increased by an increment of one and the whole estimation procedure was repeated. The

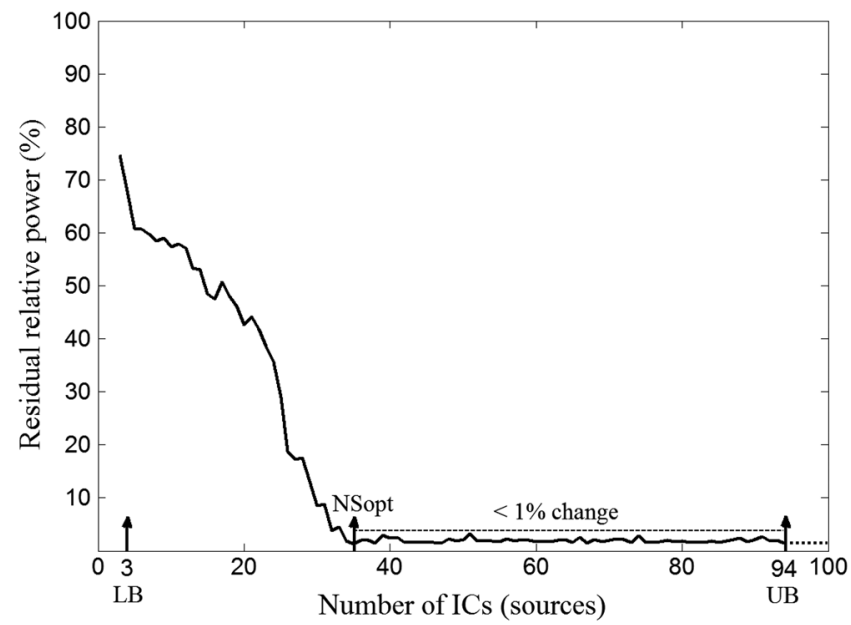

Fig. 3 Residual relative power (output of the source number estimation procedure) as a function of number of sources for Subject 1 . The curve shows how the residual relative power decreases with increasing number of sources. For this subject, only one fNIRS channel was excluded from the decomposition procedure due to its poor quality. Sources above the upper bound (94) have been rejected by PCA. $\mathrm{LB}$, lower bound; UB, upper bound; and $\mathrm{NS}_{\mathrm{opt}}$, optimal number of sources.

optimal number of ICs was finally set to the value with which the relative power of the residual of the decomposition did not change more than $1 \%$ by increasing the number of sources (Fig. 3). The main goal of this step was to retain spectral peaks with significant power; therefore, weak spectral peaks, for which no physiological relevance could be found, were not modeled in our study.

\subsection{Single-Subject Spectral Analysis}

For each subject, we first determined the association of the spectral peaks in the PSD $_{\mathrm{SA}}$ (see Sec. 2.2.3) of deoxy-Hb, oxy-Hb, and total-Hb with different physiological components. The frequency bands and their spectral peaks with highest power for LF oscillations, respiration $\left(f_{R}\right)$, and cardiac pulsations $\left(f_{C}\right)$ were identified on the average normalized PSD of the external physiological signals.

The respiratory harmonics were also identified between $f_{R}$ and $f_{C}$. We filtered the fNIRS signals above $1.5 \mathrm{~Hz}$; therefore, the cardiac harmonics were not analyzed. To identify frequency peaks associated with nonlinear cardio-respiratory couplings, we used the cross bicoherence method ${ }^{37}$ to compute the normalized bispectrum of the external respiratory and cardiac signals for each subject. For this purpose, we used the higher-order spectral analysis toolbox in MATLAB. ${ }^{38}$

For the bispectral analysis, each fNIRS time series was partitioned into 30 nonoverlapping segments considered to be the optimal number of segments required for reliable power estimation. ${ }^{37}$ The respiration self-coupling and bifrequencies due to interactions between the respiratory and cardiac systems were then identified and labeled on the normalized average PSD of the deoxy-Hb, oxy-Hb, and total-Hb signals.

For each subject, the PSD of the back-projected signals were computed channel by channel and divided into LF fluctuations including very low frequency $\left(X_{\mathrm{VLF}}, 0.01\right.$ to $\left.0.08 \mathrm{~Hz}\right)$ and $\mathrm{LF}$ $\left(X_{\mathrm{LF}}, 0.08\right.$ to $\left.0.15 \mathrm{~Hz}\right)$ oscillations, respiration $\left(X_{f_{R}}\right.$ and its 
harmonics $\left.X_{H}\right)$, and cardiac activities $\left(X_{f_{C}}\right.$ and cardio-respiratory components $\left.X_{C R}\right)$. Then, for each channel the percentage contribution of these activities were obtained by computing the power ratio of their respective frequency band to the total power of the original signal. The resulting percentage values were averaged over all channels and over those located within different cerebral regions as indicated in Fig. 1. Since each subject underwent two to three resting-state sessions, the results were averaged over the sessions for each subject.

For each subject, we also computed the percentage of residuals resulting from the PCA method $\left(R_{\mathrm{PCA}}\right)$ and the decomposition procedure $\left(R_{\mathrm{DP}}\right)$ using the optimal number of sources.

The decomposition was formulated as

$$
\begin{aligned}
Y_{\text {fnirs }}= & A_{1} \times X_{\mathrm{VLF}}+A_{2} \times X_{\mathrm{LF}}+B_{1} \times X_{f_{R}}+B_{2} \times X_{H} \\
& +C_{1} \times X_{f_{C}}+C_{2} \times X_{\mathrm{CR}}+D \times R_{\mathrm{PCA}}+E \times R_{\mathrm{DP}}
\end{aligned}
$$

where $Y_{\text {fnirs }}$ is each channel's fNIRS signal, and $A, B, C, D$, and $E$ are the related percentage contributions of the hemodynamic sources described above. To further estimate the contributions of very low frequency (VLF) and LF related to the brain autoregulatory oscillations as well as global signals driven by variations in the $\mathrm{ABP}$, for each subject, we first decomposed the externally recorded $\mathrm{ABP}$ signal using the same technique employed to decompose the resting-state fNIRS signals (see Sec. 2.2.2). Then, we computed the maximum magnitude squared coherence $\left(C_{\mathrm{VLF}}\right.$ and $\left.C_{\mathrm{LF}}\right)$ between the VLF and LF components of the ABP signals and those of fNIRS channels. The magnitude squared coherence, a function of frequency, varies between 0 and 1 . We used the function mscohere ${ }^{39}$ to investigate frequency-domain correlation between $\mathrm{ABS}$ and fNIRS signals using Welch's overlapped averaged periodogram. The maximum magnitude squared coherence values were used to estimate the percentage contribution of the VLF and LF components of the brain oscillations ( $X_{\mathrm{BA} \_ \text {VLF }}$ and $\left.X_{\mathrm{BA} \_\mathrm{LF}}\right)$ using the following equations:

$X_{\mathrm{BA} \_\mathrm{VLF}}=\left(1-C_{\mathrm{VLF}}\right) \cdot X_{\mathrm{VLF}} \quad X_{\mathrm{BA} \_\mathrm{LF}}=\left(1-C_{\mathrm{LF}}\right) \cdot X_{\mathrm{LF}}$.

In this approach, we assumed that ABP had a global effect across all fNIRS channels.

\subsection{Intersubject Variability}

We performed a PCA-based cluster analysis to probe intersubject variability in the percentage contributions of LF fluctuations, respiration, cardiac activity, and other components to the deoxy-Hb, oxy-Hb, and total-Hb signals. For this purpose, we first created a matrix $M$ of size $P \times Q$

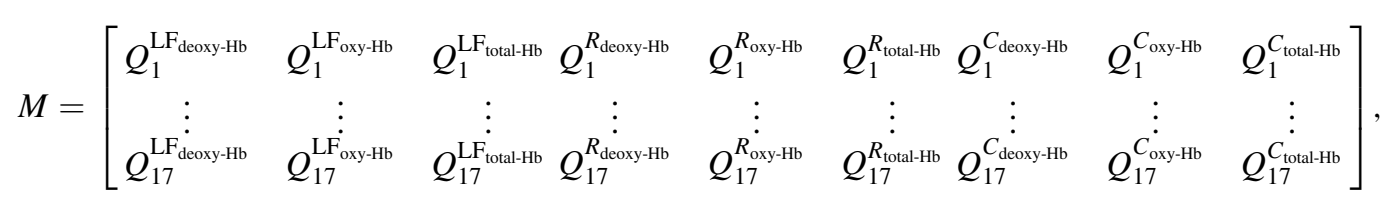

where $P$ was the total number of subjects (herein 17), and $Q$ was the total number of the average percentage contribution values obtained across all channels for the three frequency bands: LF fluctuations, respiration $(\mathrm{R})$, and cardiac activity (C) for deoxy-Hb, oxy-Hb, and total-Hb. To identify major differences between subjects, we used PCA to project the two-dimensional (2-D) matrix into a lower dimensional space. This was done before clustering to identify directions of maximal variance. ${ }^{40}$ This preprocessing has been shown to provide a more robust clustering by removing directions with low variance. We selected the first PCs, which explained at least $90 \%$ of all variations in contributions of the three frequency bands. To find a reasonable number of clusters, the reduced matrix was first clustered using the $k$-means clustering method ${ }^{41}$ with $k$ clusters. To determine the optimal number of clusters, $k$ was set to the number of selected PCs. Then, the clustering was performed and the similarity between partitions was computed. The clusters were merged if they fell inside two within-cluster standard deviation of the others. Using this approach, we found the optimal number of clusters to classify subjects based on their similarities in the percentage contributions of slow activity, respiration, and cardiac activity. Finally, we performed statistical comparison between the clusters for deoxy- $\mathrm{Hb}, \mathrm{oxy}-\mathrm{Hb}$, and total-Hb.

\section{Results}

\subsection{Spectral Analysis}

Figure 4 shows the results of the spectral analysis for Subject 1 . The normalized PSDs of the deoxy-Hb, oxy-Hb, and total-Hb signals were computed by averaging the PSDs of all the channels. In Fig. 4(a), three main peaks can be seen at $\sim 0.02 \mathrm{~Hz}$ (slow activity), $0.13 \mathrm{~Hz}$ (respiration), and $1.18 \mathrm{~Hz}$ (cardiac pulsations). The magnitudes of these peaks have obscured the other spectral peaks. To extract other spectral peaks, we used the scICA-based decomposition approach described in Sec. 2.2.2. Figure 4(b) shows the normalized PSD resulting from the decomposition of the deoxy-Hb, oxy-Hb, and total-Hb signals. In this subplot, the other spectral peaks are clearly distinguishable from the spectral background. It is of note that in this representation the magnitudes of peaks carry no information because the amplitudes of their corresponding independent components have been normalized to unity during the decomposition process.

The same spectral analysis and decomposition procedure were applied to the auxiliary signals recorded synchronously with the fNIRS signals. As shown in Figs. 4(c) and 4(d), the same spectral peaks were found for the auxiliary as those observed for the fNIRS signals. On average across all subjects, the frequency of the systemic ABP varied between 0.05 and $0.15 \mathrm{~Hz}$. The respiration and cardiac activity exhibited a single peak in the frequency ranges $[0.13$ to $0.36 \mathrm{~Hz}]$ and $[0.75$ to $1.3 \mathrm{~Hz}$, respectively. 

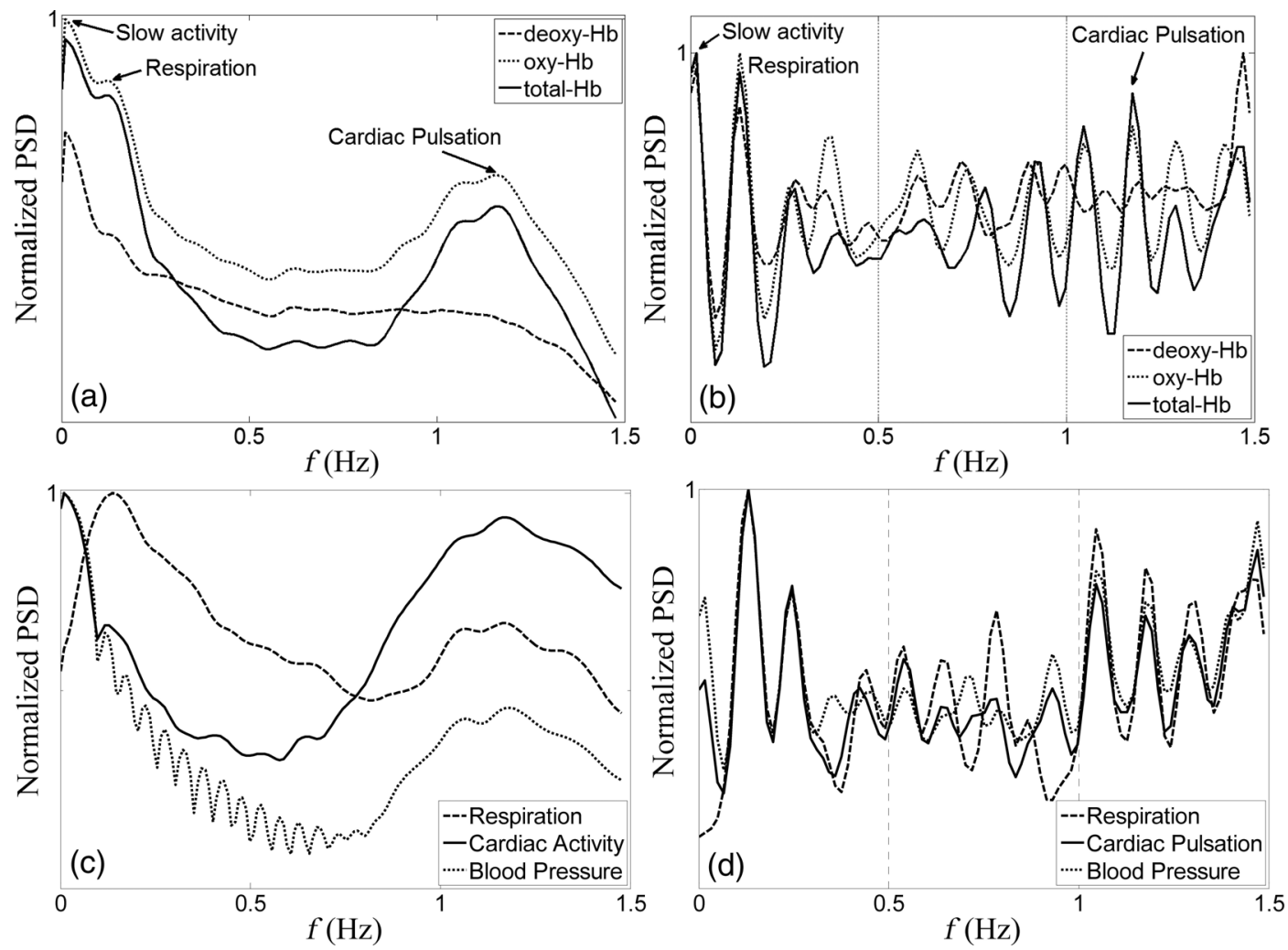

Fig. 4 Results of power spectral analysis for Subject 1. (a) Normalized PSD and (b) full spectra averaged over all source-detector pairs for deoxy- $\mathrm{Hb}$, oxy- $\mathrm{Hb}$, and total- $\mathrm{Hb}$ signals. To obtain the results in plot (a), the PSD of the deoxy- $\mathrm{Hb}$, oxy- $\mathrm{Hb}$, and total- $\mathrm{Hb}$ signals were obtained by averaging the PSDs over all the fNIRS channels. The resulting averaged PSDs were normalized to the maximum power value obtained across deoxy- $\mathrm{Hb}$, oxy- $\mathrm{Hb}$, and total- $\mathrm{Hb}$. To obtain the results in plot (b), each of the fNIRS channels was first decomposed using the single-channel decomposition method (see Sec. 2.2.2). The PSDs of the channel's independent components were normalized to their total power, and then averaged to obtain the average normalized PSD for the channel. Finally, the averaged PSDs were calculated across channels. In a similar way, plots (c and d) were obtained for the externally recorded ABP, respiration, and cardiac signals for Subject 1.

As described earlier, we also investigated nonlinear cardiorespiratory couplings using the cross-bicoherence method (Sec. 2.3) for each subject. Figure 5 shows a contour plot of the estimated bicoherence for Subject 1. As shown, the cross-bicoherence method revealed a peak at bifrequency $(0.13$ to $0.13 \mathrm{~Hz})$ indicating the respiratory self-coupling. The cardio-respiratory coupling has caused a strong bispectral peak at bifrequency $(0.13$ and $1.18 \mathrm{~Hz})$. All other peaks are related to the nonlinear couplings between the respiratory components (main peak $f_{R}$, and its harmonics $H_{1}$ and $H_{2}$ ) and the cardiac frequency $\left(f_{C}\right)$. The nonlinear interactions appear as $f_{C} \pm n \cdot f_{R}$, where $n$ refers to the $n$ 'th respiratory harmonic. All the peaks labeled at the spectral and bispectral analysis stages were used to compute the percentage contribution of LF, respiratory, and cardiac activities to the fNIRS signals.

\subsection{Contribution of Functional Near-Infrared Spectroscopy Components}

On average, the decomposition was performed for all subjects with 37 components and 49 channels after removing noisy channels. Figure 6 shows the average percentage contributions of the LF, respiratory, and cardiac activities and their cross coupling components to the deoxy-Hb, oxy- $\mathrm{Hb}$, and total-Hb signals.
For each subject, the averaged percentage contribution of each activity was first computed across all channels for (a) deoxy-Hb, (b) oxy-Hb, and (c) total-Hb. The grand average percentage values were then computed across all subjects. As shown in Figs. 6(a)-6(c), slices 1 and 2, during the resting period two LF components (below $0.15 \mathrm{~Hz}$ ) were observed. One peaked between 0.01 and $0.08 \mathrm{~Hz}$, associated with slow activities including spontaneous brain hemodynamic activities as well as very LF content of blood pressure variations [shown as VLF in Fig. 6(d)], and the other peaked between 0.08 and $0.15 \mathrm{~Hz}$, mainly related to variations in blood pressure represented as LF in Fig. 6(d). The percentage contributions of VLF were much higher than those of LF in deoxy-Hb, oxy-Hb, and total-Hb.

The main spectral peak of the respiration (Fig. 6, slice and peak 3) showed a higher contribution to deoxy- $\mathrm{Hb}(9.4 \%)$ in comparison with the one computed for the spectral peak of the cardiac activity (7.3\%; Fig. 6, slice and peak 5 and Table 1). An inverse trend was observed for oxy-Hb and total-Hb. Interestingly, compared to oxy- $\mathrm{Hb}(7.2 \%)$ and total$\mathrm{Hb}(5.9 \%)$, deoxy-Hb (10.9\%) showed higher contributions for the respiratory harmonics (Fig. 6, slice and peak 3) as well as for the nonlinear cardio-respiratory couplings (Fig. 6, slice and peak 6 and Table 1). No significant differences 


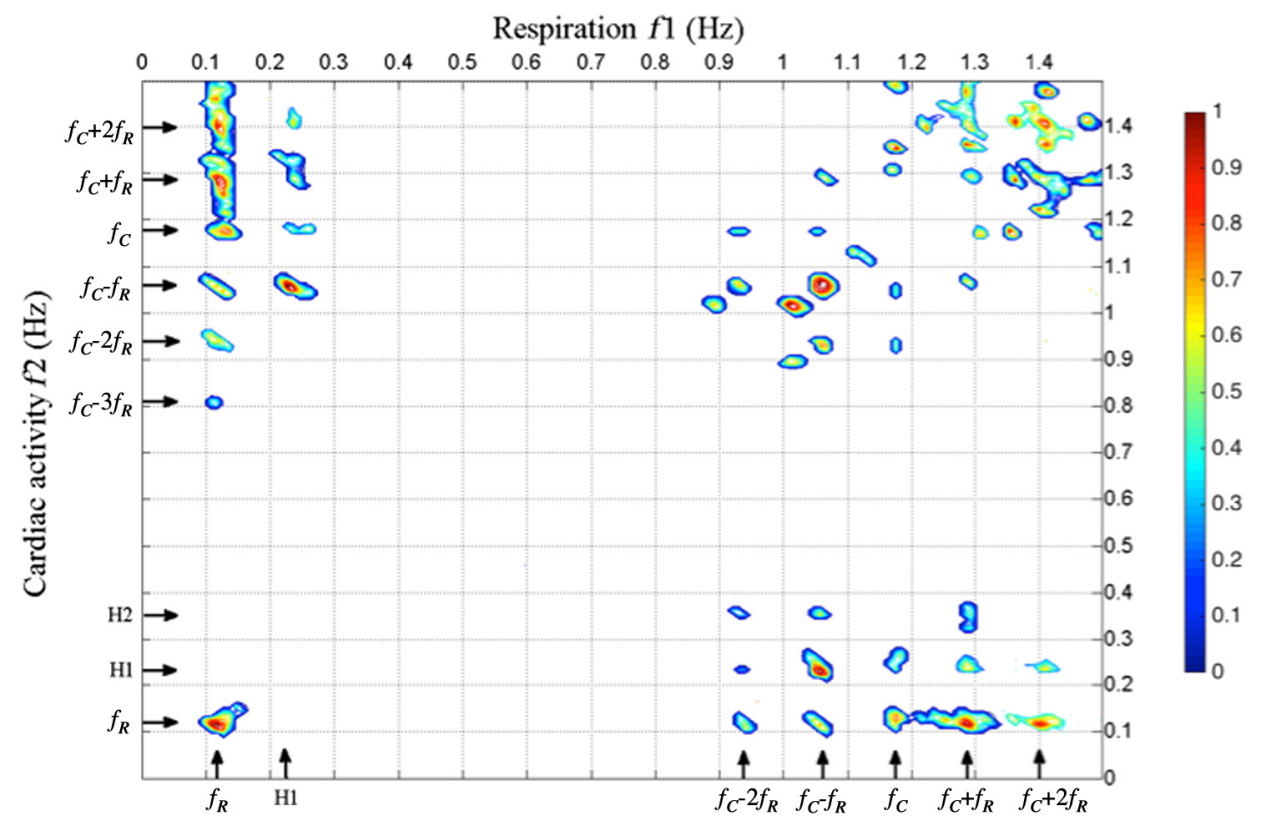

Fig. 5 Results of the bispectral analysis between the respiratory and cardiac signals for Subject 1. As shown, the cross-bicoherence analysis reveals phase coupling between the respiratory and cardiac systems. The strong peak at bifrequency $(0.13$ and $0.13 \mathrm{~Hz})$ indicates the respiratory self-coupling. The peak at bifrequency $(0.13$ and $1.18 \mathrm{~Hz})$ is related to the cardio-respiratory coupling. All other peaks $\left(f_{2} \pm f_{1}\right)$ belong to the nonlinear couplings between the respiratory components (main peak $f_{B}$, and its harmonics $H_{1}$ and $H_{2}$ ) and the main peak of the cardiac activity $\left(f_{C}\right)$. The color scale is shown normalized to the maximum peak in the image.
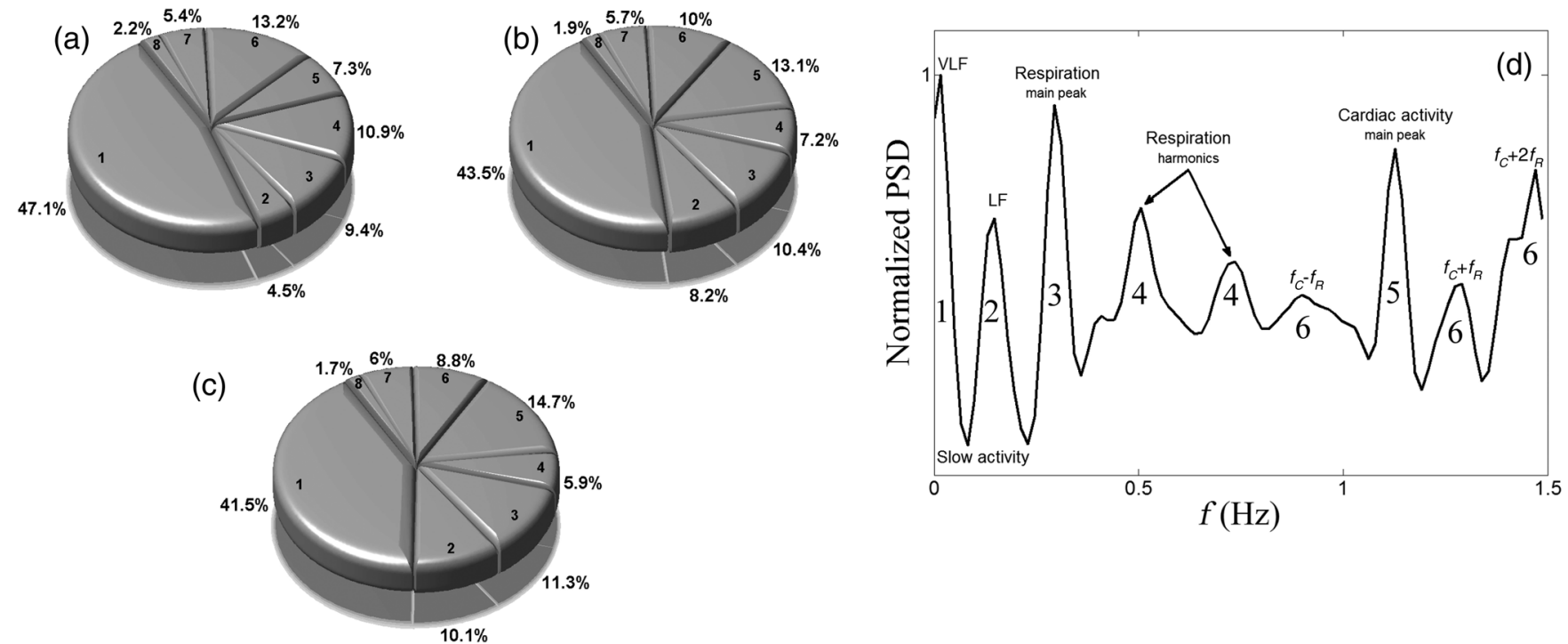

Fig. 6 Average percentage contribution of LF, respiratory, and cardiac activities to the deoxy-Hb (panel a), oxy-Hb (panel b), and total-Hb (panel c) signals. The pie charts show the contributions of LF activities (slices 1 and 2), respiration (slice 3 ) and its harmonics (slice 4), cardiac activity (slice 5) and its nonlinear couplings with the respiratory components (slice 6). The percentage of residuals resulted from the PCA method (slice 7) and that from our decomposition method (slice 8) have been also shown in the pie charts (a)-(c). The slices 1 to 6 correspond to peaks 1 to 6 shown in plot (d).

were observed in the residuals of the PCA and decomposition methods between deoxy-Hb, oxy-Hb, and total-Hb [Figs. 6(a)6(c), slices 7 and 8 and Table 1].

The percentage contributions of the subcomponents of LF, respiration, and cardiac activities have been shown in Table 1. As illustrated, the main contributions belonged to VLF, and the main spectral peaks of respiration and cardiac activities for deoxy-Hb, oxy-Hb, and total-Hb. For slow activities, LF showed higher contributions for oxy- $\mathrm{Hb}$ and total-Hb in comparison with deoxy-Hb.

On average across all subjects, in the VLF band, almost $80 \%$ of the contributions belonged to the brain autoregulatory activities and the rest to the systemic blood pressure variations. In the LF band, more than $85 \%$ of the contributions belonged to the 
brain autoregulatory activities for deoxy-Hb, oxy-Hb, and totalHb. Averaged over subjects, the percentage contributions of the VLF components of the brain autoregulatory activity for deoxy$\mathrm{Hb}$, oxy- $\mathrm{Hb}$, and total-Hb were more than four times the values obtained for the systemic changes in the ABP signal. This ratio was much higher for LF.

Concerning the respiratory subcomponents, the main peak has shown the highest percentage contribution compared to the other subcomponents. However, the first and the second harmonics as well as the nonlinear cardio-respiratory couplings $\left(f_{C} \pm f_{R}\right)$ showed increased contributions to deoxy-Hb in comparison with those found for oxy-Hb and total-Hb. However, the main cardiac peak showed higher contribution to oxy- $\mathrm{Hb}$ and total-Hb compared to deoxy-Hb (Table 1).

Figure 7 shows the regional differences in the contributions of LF (sum of VLF and LF), respiration (sum of the main peak

Table 1 Average percentage distribution of sub-components of the slow activity, respiration, and cardiac activities. The percentage values were computed by averaging the values obtained for all subjects. See Fig. 5 for component descripion.

\begin{tabular}{|c|c|c|c|}
\hline & $\mathrm{Oxy}-\mathrm{Hb}$ & $\mathrm{Deoxy}-\mathrm{Hb}$ & Total-Hb \\
\hline Slow oscillations (total) & $51.70 \%$ & $51.60 \%$ & $51.60 \%$ \\
\hline VLF & $43.50 \%$ & $47.10 \%$ & $41.50 \%$ \\
\hline LF & $8.20 \%$ & $4.50 \%$ & $10.10 \%$ \\
\hline Respiratory (total) & $17.60 \%$ & $20.30 \%$ & $17.20 \%$ \\
\hline Main & $10.40 \%$ & $9.40 \%$ & $11.30 \%$ \\
\hline Harmonics (all) & $7.20 \%$ & $10.90 \%$ & $5.90 \%$ \\
\hline $1 s t$ & $5.78 \%$ & $7.86 \%$ & $4.74 \%$ \\
\hline $2 n d$ & $1.16 \%$ & $2.52 \%$ & $1.01 \%$ \\
\hline $3 r d$ & $0.20 \%$ & $0.44 \%$ & $0.13 \%$ \\
\hline 4 th & $0.06 \%$ & $0.07 \%$ & $0.02 \%$ \\
\hline Cardiac (total) & $23.10 \%$ & $20.50 \%$ & $23.50 \%$ \\
\hline Main & $13.10 \%$ & $7.30 \%$ & $14.70 \%$ \\
\hline Cardio-Respiratory (all) & $10.00 \%$ & $13.20 \%$ & $8.80 \%$ \\
\hline$f_{C}+f_{R}$ & $3.19 \%$ & $4.05 \%$ & $2.97 \%$ \\
\hline$f_{C}-f_{R}$ & $4.94 \%$ & $6.15 \%$ & $4.28 \%$ \\
\hline$f_{C}+2 f_{R}$ & $0.88 \%$ & $1.34 \%$ & $0.64 \%$ \\
\hline$f_{C}-2 f_{\mathrm{R}}$ & $0.56 \%$ & $0.90 \%$ & $0.44 \%$ \\
\hline$f_{C}+3 f_{R}$ & $0.32 \%$ & $0.53 \%$ & $0.27 \%$ \\
\hline$f_{C}-3 f_{R}$ & $0.12 \%$ & $0.22 \%$ & $0.20 \%$ \\
\hline Noise (total) & $7.60 \%$ & $7.60 \%$ & $7.70 \%$ \\
\hline Unaccounted physiology & $5.70 \%$ & $5.40 \%$ & $6.00 \%$ \\
\hline White noise & $1.90 \%$ & $2.20 \%$ & $1.70 \%$ \\
\hline Total & $100.00 \%$ & $100.00 \%$ & $100.00 \%$ \\
\hline
\end{tabular}

and its harmonics), and cardiac activity (sum of the main peak and its nonlinear cardio-respiratory couplings) to the deoxy-Hb, oxy- $\mathrm{Hb}$, and total-Hb signals. To obtain these results, we computed the mean and standard deviation of the contribution values across all channels positioned in the left and right frontal, temporal, parietal, and occipital regions (see Fig. 1) across all subjects. We then statistically compared different cerebral regions using the Mann-Whitney nonparametric test ${ }^{42}$ with $P<0.05$.

For deoxy-Hb, statistical comparisons showed significant bilateral decrease in the contribution of the brain slow activity (VLF) moving from the anterior to the posterior parts of the brain. Conversely, the cardiac activity showed higher contributions in the posterior parts of the brain. The right frontal and temporal lobes showed higher percentage contribution for the slow activity with respect to the left side. In all other cases, symmetry was observed between the left and right hemisphere of the brain. In oxy-Hb and total-Hb signals, differences were statistically significant between the occipital regions and frontal areas for LF brain activities and cardiac pulsations. Higher variations were observed in the power of the cardiac activity in the posterior regions across all subjects. For respiration, no significant differences between cerebral regions were observed in deoxy-Hb, oxy-Hb, and total-Hb signals.

\subsection{Intersubject Variability}

Figure 8 shows the results of the intersubject clustering. Using the clustering analysis, we identified two distinct physiology types (denoted I and II) included nine and eight subjects, respectively. There were two primary features that distinguished the two physiology types. First, type-I showed a lower contribution of slow activity including VLF and LF components to the deoxy-Hb, oxy-Hb, and total-Hb signals in comparison with type-II. Second, the type-I cluster contained a higher percentage contribution for the respiration (main peak and its harmonics) and cardiac activity (main peak and nonlinear coupling components) in comparison to type-II. Table 2 summarizes all significant differences between physiology type-I and type-II with $P<0.05$.

We further used the unpaired $t$-test with a significance level of $P<0.05$ to determine whether the frequencies of the main peak for slow activity, respiration, and cardiac activities differed significantly between the two subject groups. The subjects exhibiting physiology type-I had significantly lower frequencies for slow activity $(0.012 \pm 0.002 \mathrm{~Hz})$ and respiration $(0.21 \pm 0.05 \mathrm{~Hz})$ in comparison with the second subject group showing physiology type-II with $(0.014 \pm 0.004 \mathrm{~Hz})$ for slow activity and $(0.27 \pm 0.05 \mathrm{~Hz})$ for respiration. No significant differences in cardiac frequencies $(1.07 \pm 0.15 \mathrm{~Hz})$ were found between both physiology types.

For each physiology type, Fig. 9 shows the contribution ratio (oxy-Hb/deoxy-Hb) for slow activity, respiration, and cardiac activity per subject. As shown, for slow activity and respiration, subjects characterized by type-I physiology exhibited slightly higher contribution ratios in comparison with type-II. However, for type-II, the cardiac contribution ratios were greater than those observed for type-I.

\section{Discussion}

We have presented a method for decomposing fNIRS signals based on the information from the temporal and spectral domains. The fNIRS data were collected during the resting state across the whole brain in the frontal, temporal, parietal, 

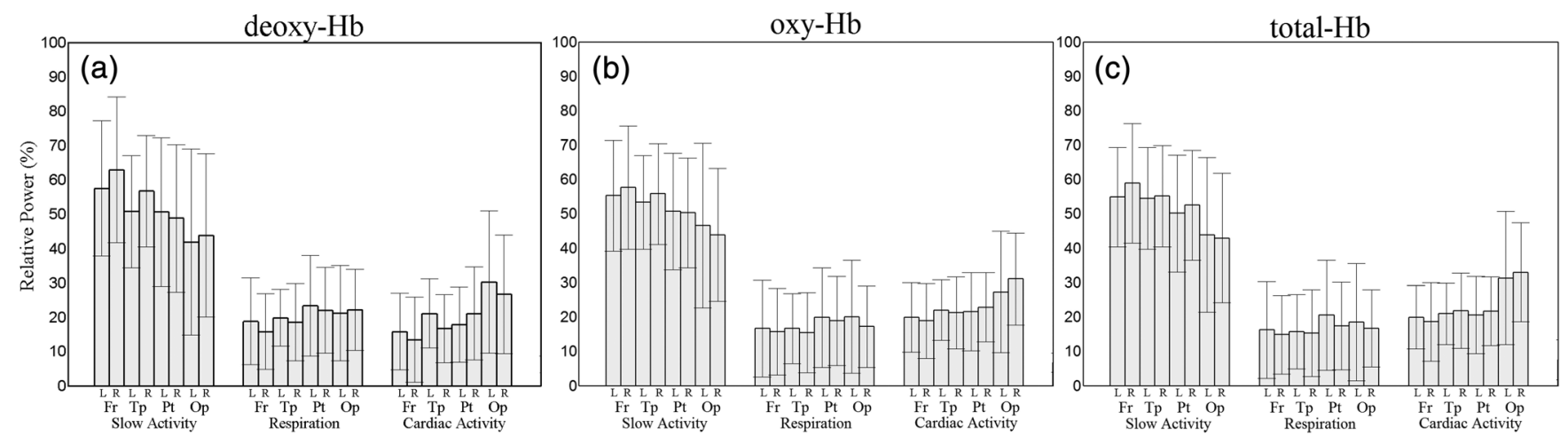

Fig. 7 Spatial distribution of contribution of slow activity, respiration, and cardiac activity to the (a) deoxy$\mathrm{Hb}$, (b) oxy-Hb, and (c) total- $\mathrm{Hb}$ signals. The results are represented as the mean and standard deviation of percentage contribution values computed over the fNIRS channels positioned in the left $(L)$ and right $(\mathrm{R})$ frontal $(\mathrm{Fr})$, temporal $(\mathrm{Tp})$, parietal $(\mathrm{Pt})$, and occipital $(\mathrm{Op})$ regions across all subjects.

\section{Physiology type-I}
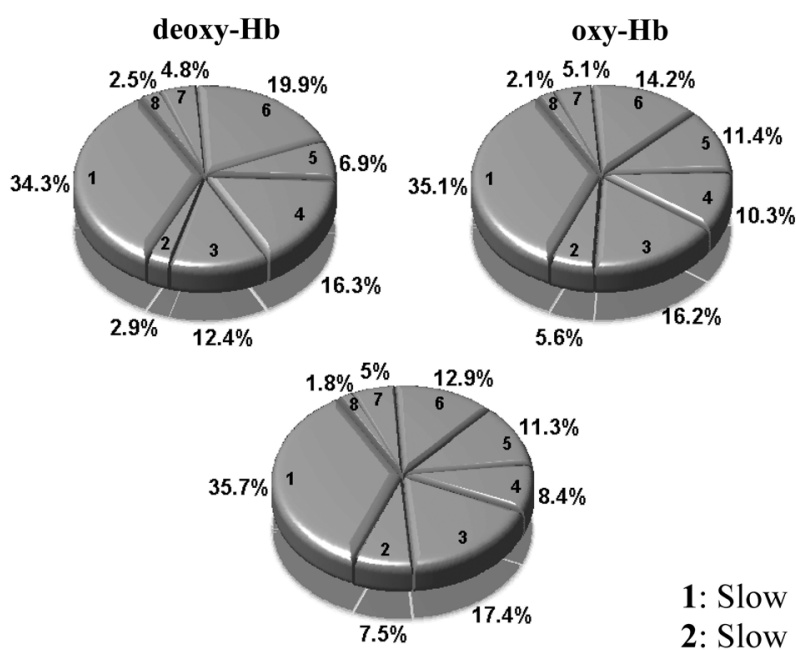

total-Hb
1: Slow activity $\sim[0.01-0.08 \mathrm{~Hz}]$

2: Slow activity $\sim[0.08-0.15 \mathrm{~Hz}]$

3: Respiration $\sim[0.13-0.36 \mathrm{~Hz}]$

4: Respiration harmonics

5: Cardiac activity $\sim[0.75-1.3 \mathrm{~Hz}]$

6: Cardio-respiratory couplings

7: Resuduals from PCA

8: Residuals from the decomposition method

\section{Physiology type-II}
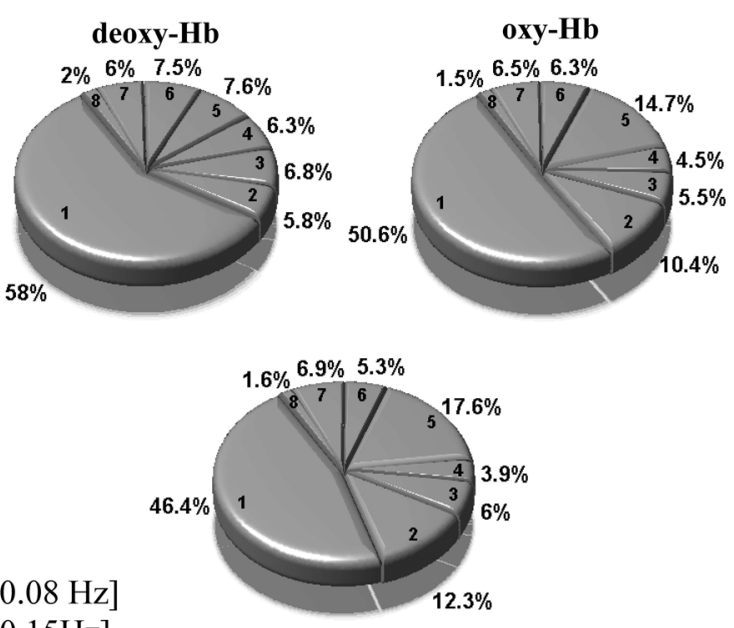

total-Hb

Fig. 8 Results of the intersubject clustering performed on the percentage contribution of slow activity, respiration, and cardiac activities to the deoxy- $\mathrm{Hb}$, oxy- $\mathrm{Hb}$, and total- $\mathrm{Hb}$ signals.

and occipital locations. The multichannel optical data were first converted to deoxy-Hb and oxy-Hb signals and then preprocessed to remove motion artifacts and noisy channels. The multichannel deoxy-Hb, oxy-Hb, and total-Hb signals were analyzed in the spectral domain to obtain an average normalized spectrum across all fNIRS channels. The full spectrum was then used to decompose fNIRS signals through the single-channel ICA method on a channel-by-channel basis. The fNIRS signal was decomposed into components that incorporated information of interest from the spatial, temporal, and the spectral domain. The components of interest contained nonredundant information with no overlap in the frequency domain. The independent components were regrouped based on their frequency spectral similarities to identify different spatio-temporal components that contributed to the deoxy-Hb, oxy-Hb, and total-Hb signals. The results were then explored to investigate the spatial variability across unilateral and bilateral brain areas in all subjects and consequently to estimate the contribution of LF oscillation, respiration, and cardiac pulsations to the deoxy$\mathrm{Hb}$, oxy- $\mathrm{Hb}$, and total- $\mathrm{Hb}$ signals. For group inference, the results of the scICA analysis performed on each subject were compared.

\subsection{Single-Channel Decomposition}

Previous approaches to removing contamination from systemic physiology on fNIRS data have fallen into either spatial or temporal methods. Spatial methods such as $\mathrm{PCA}^{9,43}$ or spatial ICA 
Table 2 Characteristic significant differences between Clusters 1 and 2 (physiology type I and II). VLF, very low frequency; LF, low frequency; $f_{R}$, respiration, main peak; $H$, respiration, harmonics; $f_{C}$, cardiac activity, main peak; $f_{C} \pm n \cdot f_{R}$, cardio-respiratory couplings; and NS, not significant.

Cluster $1(\mathrm{C} 1)$ versus Cluster $2(\mathrm{C} 2)$

\begin{tabular}{|c|c|c|c|c|}
\hline \multirow[b]{2}{*}{ Activity } & \multirow[b]{2}{*}{ Component } & & & \\
\hline & & deoxy-Hb & oxy-Hb & total- $\mathrm{Hb}$ \\
\hline \multirow[t]{2}{*}{ Slow activity } & VLF & $\mathrm{C} 1<\mathrm{C} 2$ & $\mathrm{C} 1<\mathrm{C} 2$ & $\mathrm{C} 1<\mathrm{C} 2$ \\
\hline & LF & $\mathrm{C} 1<\mathrm{C} 2$ & $\mathrm{C} 1<\mathrm{C} 2$ & $\mathrm{C} 1<\mathrm{C} 2$ \\
\hline \multirow[t]{2}{*}{ Respiration } & $f_{R}$ & $\mathrm{C} 1>\mathrm{C} 2$ & $\mathrm{C} 1>\mathrm{C} 2$ & $\mathrm{C} 1>\mathrm{C} 2$ \\
\hline & $H$ & $\mathrm{C} 1>\mathrm{C} 2$ & $\mathrm{C} 1>\mathrm{C} 2$ & $\mathrm{C} 1>\mathrm{C} 2$ \\
\hline \multirow[t]{2}{*}{ Cardiac activity } & $f_{C}$ & $\mathrm{C} 1<\mathrm{C} 2$ & $\mathrm{C} 1<\mathrm{C} 2$ & $\mathrm{C} 1<\mathrm{C} 2$ \\
\hline & $f_{C} \pm n \cdot f_{R}$ & $\mathrm{C} 1>\mathrm{C} 2$ & $\mathrm{C} 1>\mathrm{C} 2$ & $\mathrm{C} 1>\mathrm{C} 2$ \\
\hline Cluster & Component & deoxy-Hb & oxy-Hb & total-Hb \\
\hline \multirow[t]{5}{*}{1} & $\left\{f_{R}\right\}$ versus $\{H\}$ & $<$ & $>$ & $>$ \\
\hline & $\left\{f_{C}\right\}$ versus $\left\{f_{C} \pm n \cdot f_{R}\right\}$ & $<$ & $<$ & $<$ \\
\hline & $\left\{f_{R}\right\}$ versus $\left\{f_{C}\right\}$ & $>$ & $>$ & $>$ \\
\hline & $\{H\}$ versus $\left\{f_{C} \pm n \cdot f_{R}\right\}$ & $<$ & $<$ & $<$ \\
\hline & $\left\{f_{R}+H\right\}$ versus $\left\{f_{C}+\left(f_{C} \pm n \cdot f_{R}\right)\right\}$ & $>$ & $>$ & $>$ \\
\hline \multirow[t]{5}{*}{2} & $\left\{f_{R}\right\}$ versus $\{H\}$ & NS & $>$ & $>$ \\
\hline & $\left\{f_{C}\right\}$ versus $\left\{f_{C} \pm n \cdot f_{R}\right\}$ & NS & $>$ & $>$ \\
\hline & $\left\{f_{R}\right\}$ versus $\left\{f_{C}\right\}$ & $<$ & $<$ & $<$ \\
\hline & $\{H\}$ versus $\left\{f_{C} \pm n \cdot f_{R}\right\}$ & $<$ & $<$ & $<$ \\
\hline & $\left\{f_{R}+H\right\}$ versus $\left\{f_{C}+\left(f_{C} \pm n \cdot f_{R}\right)\right\}$ & $<$ & $<$ & $<$ \\
\hline
\end{tabular}

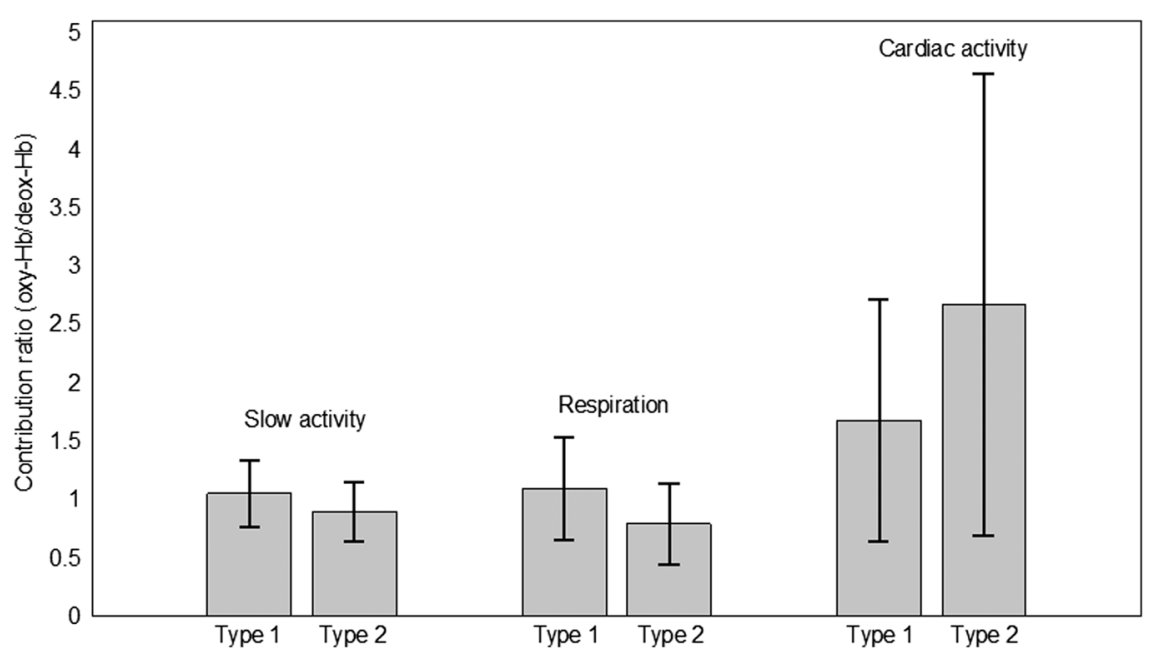

Fig. 9 Contribution ratio (oxy-Hb/deoxy-Hb) for slow activity, respiration, and cardiac activities per subject and physiology type.

methods attempt to remove systemic physiology by projecting out components with the same spatial covariance as systemic noise from the measurements of interest. For example, in the work by Franceschini et al., ${ }^{11}$ a resting (baseline) scan was used to construct the spatial components of systemic noise from PCA, which were then projected from the fNIRS scans during the task. However, this approach works well only when the several conditions are met. First, the spatial covariance of the task-related activity must be distinguishable from the background systemic noise. In practice, this requires a large 
number of fNIRS measurements that cover an area of the head larger then the region activated by the task. Second, the spatial covariance of the physiological noise signal must be stationary. Finally, this approach requires that measurement channels with poor coupling to the head (e.g., high levels of random noise) be removed in preprocessing to accurately estimate the spatial covariance structures. While spatial filtering methods can be applied to analysis of task-related fNIRS signals, these methods cannot be applied to resting-state connectivity studies.

Temporal filtering methods have also been proposed for removing systemic noise. For removal of high-frequency noise such as the main frequency of the cardiac cycle, lowpass linear filtering methods can be applied. However, conventional bandpass filtering will not work properly to remove respiratory, heart-rate variability, or blood pressure-induced fluctuations mainly because the frequency band of interest to be removed is not known a priori and in general are not well separated spectrally from the frequencies of the evoked hemodynamic brain response. Temporal regression methods have been used to remove systemic noise, but require a measure of external physiology with a good quality to remove physiological noise. These methods provide undesirable results when the transfer functions between the sources of activities (here heart and lungs) and the brain are time varying or nonlinear. Diamond et al. ${ }^{44}$ proposed an adaptive regression method using a Kalman filter to address this issue of stationarity of the transfer function from systemic measurements to the brain signal. However, the assumption of a nonstationary linear transfer function from systemic circulation to the brain is an oversimplification of the system. Alternatively, fNIRS measurements with a short separation between the source and detector positions can provide a more regional estimate of systemic physiology since these measurements only probe the superficial skin layers. ${ }^{6}$ These short distances can be used as a local regressor of physiological noise. However, these short-separation signals represent a mixture of different physiological contributions (cardiac, respiratory, and blood pressure) that cannot be separated. When these measurements are used as a linear regressor, an assumption is made that the relative contributions of the physiological signals within the regressor are the same as the contamination in the fNIRS brain measurement. This is only partially true since the deeper measurements contain contamination from both the skin and brain and the brain may have a quite different sampling of arterial and venous contributions from what is captured in the short-distance measurement. When multiple physiological oscillations are contained within a single regressor as is the case in the short-distance correction methods, there is a possibility to introduce artifact frequencies into the measurement of interest, e.g., if ratio of arterial-venous sensitivity differs from the regressor. This is also true in the case of using measurements of systemic physiology from the body. For example, a pulse-oximeter signal from the finger contains a mixture of both cardiac (and harmonics) and respiratory (and harmonics) frequencies. Across the brain, the ratio of these mixed components will vary.

Single-channel ICA is a demixing procedure for decomposing a single time course into its constituent components. Unlike spatial ICA, which decomposes a matrix of data (multiple channels recorded over time) into components, scICA operates on a vector of data (single channel over time) and decomposes the data based on statistically independent components consisting of groups of covarying frequencies from within that one channel. This is an underdetermined decomposition, where a single channel is converted into multiple independent time series. For source analysis in fNIRS signals, we found several advantages for scICA over spatial ICA. First of all, if only a single fNIRS channel signal is recorded or very few channels are available, scICA is able to decompose the signal into its constituent components even with small contribution to the signal. ${ }^{33}$ In the case of multichannel recordings, scICA still performs much better than spatial ICA when the activity of interest is highly localized and present only on a few channels. This is because the scICA method utilizes temporal information to isolate underplaying components. Second, the basic assumption to use spatial ICA is that the propagation delays between sources and sensors are negligible. This is not the case for the brain hemodynamic activities with spatially varying delays. ${ }^{45}$ This property affects the identifiability power of the spatial ICA method. However, scICA produces less desirable results under circumstances where the signal generated by underlying sources has components with joint spectral support in the frequency domain. ${ }^{33}$

The success of ICA analysis is dependent on several factors. First, the output of spatial and/or temporal ICA depends on the number of components. ${ }^{46}$ If too few components are used, the signal will not be efficiently unmixed. However, if too many components are used, then the underlying signals (physiology) will be split across multiple components. In our approach, the produced components need to be analyzed to identify noiserelated components and the relevant ones related to the signal of interest. ${ }^{47}$ To solve these problems, we developed two objective algorithms to efficiently estimate the number of sources as well as to identify the components related to LF oscillations, respiration, and cardiac activity. Our approach for estimating the number of sources searched the optimal number of components between a lower bound, defined as the minimum number of components needed to identify three main frequency bands associated with LF activities, respiration, and cardiac pulsations, and a upper bound, defined as the minimum number of PCs used to reduce data dimensionality. We also used the robustICA method, which has the advantage over the commonly used fastICA algorithm, which is quite sensitive to the initial values. ${ }^{30}$ In addition, the efficiency of our decomposition approach depended on how well the components related to the respiratory harmonics as well as those related to the nonlinear cardio-respiratory couplings could be identified. To label spectral peaks, we required external physiology. This might be considered as a weakness of our method; however, we only used these reference signals to identify peaks not for the decomposition purpose.

\subsection{Contribution of Functional Near-Infrared Spectroscopy Components}

Using scICA, we were able to decompose the deoxy-Hb, oxy$\mathrm{Hb}$, and total-Hb signals into their underlying components. The benefits of our approach are twofold. First, as we showed, on average across all subjects, $93 \%$ of total fNIRS signal power could be decomposed through the decomposition procedure into the constituent components including LF oscillations, respiration and its harmonics, and cardiac pulsations and cardiorespiratory components. The remaining signal power constituted the residuals of our method and PCA. This decomposition helped us to understand the percentage contribution of different physiological sources to deoxy-Hb, oxy-Hb, and total-Hb. 
Compared to oxy-Hb and total-Hb, deoxy-Hb showed higher variability across subjects. This is consistent with the results reported by others. ${ }^{47}$ This can be explained by the lower SNR of deoxy-Hb, which made the full decomposition a bit harder. However, during the full spectrum identification process, we found that the powers of fluctuations of different frequency bands in deoxy-Hb were lower than those in oxy-Hb and total$\mathrm{Hb}$, but their percentage contribution were slightly different from oxy-Hb and total-Hb. Similar observations have been reported by Cui et al. ${ }^{48}$ In our study, as expected, oxy-Hb and total-Hb showed a higher percentage contribution in LF fluctuations and cardiac pulsations in comparison with deoxy-Hb. This is in agreement with the findings reported by other research groups. 5,49

We observed higher contribution ( $40 \%$ to $45 \%$ ) for very $\mathrm{LF}$ fluctuations $(0.01$ to $0.08 \mathrm{~Hz})$ than that $(5 \%$ to $10 \%)$ found for LF oscillations ( 0.08 to $0.15 \mathrm{~Hz}$ ). However, VLF and LF exhibited similar spatial extent.

LF fluctuations have shown a global physiological origin. ${ }^{27}$ As shown in Fig. 8, we also observed a global effect in VLF and $\mathrm{LF}$ in the deoxy-Hb, oxy-Hb, and total-Hb signals. The LF power was more pronounced in the anterior regions; however, symmetry was observed between the left and right hemispheres.

In a recent study, through the analysis of fNIRS data collected in the sensorimotor region, Katura et al. ${ }^{21}$ have shown that $35 \%$ of the oxy-Hb signal could be attributed to VLF and LF activities. As shown in Table 1, we found that higher average percentage contribution (50\%) for VLF and LF oscillations in deoxy- $\mathrm{Hb}$, oxy- $\mathrm{Hb}$, and total-Hb across all brain areas during the resting state. Duan et al. ${ }^{7}$ suggested that the LF fluctuations in resting-state fNIRS signals may reflect spontaneous brain activity. Since LF fluctuations from background physiology overlap with the LF fluctuations from neuronal activity, we therefore postulate that the increase that we observed in the power of LF fluctuations relates more to the spontaneous background physiology. This may also show that the LF content of fNIRS data decreases in task-induced brain activation.

Using fMRI, a high proportion $(75 \%)$ of the global LF blood oxygen level dependent noise variance has shown to be attributed to respiratory factors. ${ }^{50}$ In our study, the respiratory main contributions were in the main respiratory peak and its harmonics, which on average constituted $20 \%$ of the total power of the deoxy- $\mathrm{Hb}$, oxy-Hb, and total-Hb signals. In the fMRI signal, the respiratory artifact is due to both physiological blood pressure and oxygenation changes similar to those expected to effect the fNIRS signal, but also due to the magnetic susceptibility artifact from expansion of the lungs. This susceptibility artifact only affects the MRI signal and would not show up in the fNIRS and could explain some of the reason why respiratory oscillations account for a larger faction of MRI variance. In addition, fNIRS signals contain contributions from both the skin/scalp and the brain, and this might also account for a discrepancy with the fMRI reports.

\subsection{Variability Between Subjects}

On average, we found $20 \%$ variability across subjects in different frequency bands. The intersubject variability could be explained by differences between the brain hemodynamic responses of the subjects during the resting state. However, other reasons included the imprecision in the position of the optode between subjects, and removing noisy and artifactual fNIRS channels in the predecomposition stage. In some cases, the latter process reduced the number of channels per brain regions to one. This increased the variability per subject and consequently across subjects.

Our data fell into roughly two types of physiology, with type-I subjects associated with lower relative cardiac and slow component activity, but higher respiratory contributions than type-II. Since this result was for sensors placed all around the head, this finding seems to reflect global physiological patterns and could be explained by differences in the relative contribution of the arterial and venous compartments between subjects in these two groups. Physiologically, this could reflect different levels of baseline cerebral perfusion [e.g., baseline blood flow, volume, vascular volume fractions, or vascular transit time (the ratio of baseline flow and volume; see Ref. 51)] and further investigation is needed to understand these subject-level differences.

\subsection{Application of Single-Channel Independent Component Analysis to Physiological Noise Removal}

This work focused on the identification of physiological sources of noise within fNIRS data collected from a probe covering the whole head. A motivation for this study to better understand the nature of this noise is the aim to improve the estimate of brain activity or to characterize neural-related resting-state fluctuations. Using single-channel ICA, we were able to decompose the time course of a single fNIRS measurement into its constituent physiological components. We propose that this decomposition could be used in future work in the context of removing physiology from fNIRS measurements in several ways. First, following the spatial-temporal methods previously described in this context based on conventional PCA or ICA such as those described in Zhang et al.,, ${ }^{9,43}$ the scICA method could allow more control over removing subcomponents of the signals. In addition, this method could be applied to filter shortseparation fNIRS signals wherein the short-separation measurements, which correspond to local scalp measurements, could be further decomposed by scICA and used as multiple regressors. In the short-separation regression methods (e.g., White et al. ${ }^{6}$ ), the measurement of the scalp signal is used to regress noise from the longer-separation measurements to isolate the brain from the scalp. However, this regression assumes that the relative contributions of the arterial/venous compartments are the same across the probe volume. In other words, a single coefficient is used to model the relationship of the total signal (cardiac, blood pressure, and respiratory) in the short-separation compared to the long-separation measurements. The scICA method applied to the short-separation measurement could provide the means to unmix these physiological components, which could allow multiple regressors to be used in the filtering to account for differences in the relative contributions of these signals across space. Future work is needed to investigate this hypothesis.

\section{Conclusion}

In summary, our study makes several contributions to the fNIRS analysis field. First, the proposed approach decomposes fNIRS measurements into their underlying components or sources. It also provides information on the number of sources, their spatial distribution, as well as their temporal and spectral content. Most importantly, this approach can be applied to single-channel fNIRS data as well as the recordings with multiple channels. The decomposition method is not sensitive to the spatially varying propagation delays between sensors and the sources of activities. We 
investigated the percentage contribution of the systemic physiology to the deoxy- $\mathrm{Hb}$, oxy- $\mathrm{Hb}$, and total-Hb signals from subjects in the resting state. Our results enable us to better understand the brain hemodynamics during the resting state.

\section{Acknowledgments}

This work was supported by the National Institutes of Health (TJH- NIH-RO1EB013210). The authors thank Maria Angela Franceschini and David Boas for providing the data used in this study.

\section{References}

1. F. F. Jobsis, "Noninvasive, infrared monitoring of cerebral and myocardial oxygen sufficiency and circulatory parameters," Science 198, 1264 1267 (1977).

2. A. Villringer et al., "Near infrared spectroscopy (NIRS): a new tool to study hemodynamic changes during activation of brain function in human adults," Neurosci. Lett. 154, 101-104 (1993).

3. M. Cope et al., "Methods of quantitating cerebral near infrared spectroscopy data," Adv. Exp. Med. Biol. 222, 183-189 (1988).

4. M. Ferrari and V. Quaresima, "A brief review on the history of human functional near-infrared spectroscopy (fNIRS) development and fields of application," Neuroimage 63, 921-935 (2012).

5. D. A. Boas, A. M. Dale, and M. A. Franceschini, "Diffuse optical imaging of brain activation: approaches to optimizing image sensitivity, resolution, and accuracy," Neuroimage 23(Suppl. 1), S275-S288 (2004).

6. B. R. White et al., "Resting-state functional connectivity in the human brain revealed with diffuse optical tomography," Neuroimage 47, 148156 (2009).

7. L. Duan, Y. J. Zhang, and C. Z. Zhu, "Quantitative comparison of resting-state functional connectivity derived from fNIRS and fMRI: a simultaneous recording study," Neuroimage 60, 2008-2018 (2012).

8. R. C. Mesquita, M. A. Franceschini, and D. A. Boas, "Resting state functional connectivity of the whole head with near-infrared spectroscopy," Biomed. Opt. Express 1, 324-336 (2010).

9. T. J. Huppert et al., "HomER: a review of time-series analysis methods for near-infrared spectroscopy of the brain," Appl. Opt. 48, D280-D298 (2009).

10. P. Smielewski et al., "Clinical evaluation of near-infrared spectroscopy for testing cerebrovascular reactivity in patients with carotid artery disease," Stroke 28, 331-338 (1997).

11. M. A. Franceschini et al., "Diffuse optical imaging of the whole head," J. Biomed. Opt. 11, 054007 (2006).

12. R.M. Birn, "The role of physiological noise in resting-state functional connectivity," Neuroimage 62(2), 864-870 (2012).

13. M. Izzetoglu et al., "Motion artifact cancellation in NIR spectroscopy using Wiener filtering," IEEE Trans. Biomed. Eng. 52, 934-938 (2005).

14. H. Sato et al., "Wavelet analysis for detecting body-movement artifacts in optical topography signals," Neuroimage 33, 580-587 (2006).

15. F. C. Robertson, T. S. Douglas, and E. M. Meintjes, "Motion artifact removal for functional near infrared spectroscopy: a comparison of methods," IEEE Trans. Biomed. Eng. 57, 1377-1387 (2010).

16. S. G. Diamond, K. L. Perdue, and D. A. Boas, "A cerebrovascular response model for functional neuroimaging including dynamic cerebral autoregulation," Math Biosci. 220, 102-117 (2009).

17. S. Chatterjee and A. S. Hadi, Regression Analysis by Example, 4th ed., Wiley-Interscience, Hoboken, New Jersey (2006).

18. B. W. Zeff et al., "Retinotopic mapping of adult human visual cortex with high-density diffuse optical tomography," Proc. Natl. Acad. Sci. U. S. A. 104, 12169-12174 (2007).

19. C. B. Akgul, A. Akin, and B. Sankur, "Extraction of cognitive activityrelated waveforms from functional near-infrared spectroscopy signals," Med. Biol. Eng. Comput. 44, 945-958 (2006).

20. M. Fukunaga et al., "Metabolic origin of BOLD signal fluctuations in the absence of stimuli," J. Cereb. Blood Flow Metab. 28, 1377-1387 (2008).

21. T. Katura et al., "Quantitative evaluation of interrelations between spontaneous low-frequency oscillations in cerebral hemodynamics and systemic cardiovascular dynamics," Neuroimage 31, 15921600 (2006).

22. R. Zhang et al., "Autonomic neural control of dynamic cerebral autoregulation in humans," Circulation 106, 1814-1820 (2002).

23. M. A. Franceschini et al., "Cerebral hemodynamics measured by nearinfrared spectroscopy at rest and during motor activation," in Proc. Optical Society of America In Vivo Optical Imaging Workshop, pp. 73-80, Optical Society of America (2000).

24. Y. Hoshi and M. Tamura, "Fluctuations in the cerebral oxygenation state during the resting period in functional mapping studies of the human brain," Med. Biol. Eng. Comput. 35, 328-330 (1997).

25. M. E. Davies and C. J. James, "Source separation using single channel ICA," Signal Process. 87, 1819-1832 (2007).

26. G. Strangman, M. A. Franceschini, and D. A. Boas, "Factors affecting the accuracy of near-infrared spectroscopy concentration calculations for focal changes in oxygenation parameters," Neuroimage 18, 865-879 (2003).

27. Y. Tong, L. M. Hocke, and B. Frederick, "Isolating the sources of widespread physiological fluctuations in functional near-infrared spectroscopy signals," J. Biomed. Opt. 16, 106005 (2011).

28. A. Hyvarinen and E. Oja, "Independent component analysis: algorithms and applications," Neural Netw. 13, 411-430 (2000).

29. H. Santosa et al., "Noise reduction in functional near-infrared spectroscopy signals by independent component analysis," Rev. Sci. Instrum. 84, 073106 (2013).

30. V. Zarzoso and P. Comon, "Robust independent component analysis by iterative maximization of the kurtosis contrast with algebraic optimal step size," IEEE Trans. Neural Netw. 21, 248-261 (2010).

31. N. Selvaraj et al., "Statistical approach for the detection of motion/noise artifacts in Photoplethysmogram," Conf. Proc. IEEE Eng. Med. Biol. Soc. 2011, 4972-4975, IEEE, Boston (2011).

32. A. M. Chiarelli et al., "A kurtosis-based wavelet algorithm for motion artifact correction of fNIRS data," Neuroimage 112, 128-137 (2015).

33. A. Jimenez-Gonzalez and C. J. James, "Extracting sources from noisy abdominal phonograms: a single-channel blind source separation method," Med. Biol. Eng. Comput. 47, 655-664 (2009).

34. L. Trefethen and D. Bau, Numerical Linear Algebra, Society for Industrial and Applied Mathematics, Philadelphia, Pennsylvania (1997).

35. G. R. Naik and D. K. Kumar, "Estimation of independent and dependent components of non-invasive EMG using fast ICA: validation in recognising complex gestures," Comput. Meth. Biomech. Biomed. Eng. 14, 1105-1111 (2011).

36. P. D. Welch, "The use of fast Fourier transform for the estimation of power spectra: a method based on time averaging over short, modified periodograms," IEEE Trans. Audio Electroacoust. 15, 70-73 (1967).

37. J. Jamsek, A. Stefanovska, and P. V. McClintock, "Nonlinear cardio-respiratory interactions revealed by time-phase bispectral analysis," Phys. Med. Biol. 49, 4407-4425 (2004).

38. A. Swami, J. M. Mendel, and C. L. M. Nikias, "Higher-order spectral analysis toolbox," Mathworks, 2003, http://www.mathworks.com/ matlabcentral/fileexchange/3013-hosa-higher-order-spectral-analysistoolbox (31 May 2016).

39. The Mathworks, "Magnitude-squared coherence," http://www.mathworks. com/help/signal/ref/mscohere.html (31 May 2016).

40. A. Ben-Hur and I. Guyon, "Detecting stable clusters using principal component analysis," Methods Mol. Biol. 224, 159-182 (2003).

41. P. Tan, M. Steinbach, and V. Kumar, "Cluster analysis: Basic concepts and algorithms," in Introduction to Data Mining, Vol. 1, Pearson Addison Wesley, Boston (2006).

42. H. Mann and D. Whitney, "On a test of whether one of two random variables is stochastically larger than the other," Ann. Math. Stat. 18, 50-60 (1947).

43. Y. Zhang et al., "Eigenvector-based spatial filtering for reduction of physiological interference in diffuse optical imaging," J. Biomed. Opt. 10, 011014 (2005).

44. S. G. Diamond et al., "Physiological system identification with the Kalman filter in diffuse optical tomography," Med. Image Comput. Comput. Assist. Interv. 8, 649-656 (2005).

45. Z. S. Saad et al., "Analysis and use of FMRI response delays," Hum. Brain Mapp. 13, 74-93 (2001).

46. M. D. Fox and M. E. Raichle, "Spontaneous fluctuations in brain activity observed with functional magnetic resonance imaging," Nat. Rev. Neurosci. 8, 700-711 (2007). 
47. H. Zhang et al., "Functional connectivity as revealed by independent component analysis of resting-state fNIRS measurements," Neuroimage 51, 1150-1161 (2010).

48. X. Cui et al., "A quantitative comparison of NIRS and fMRI across multiple cognitive tasks," Neuroimage 54, 2808-2821 (2011).

49. M. A. Franceschini et al., "Hemodynamic evoked response of the sensorimotor cortex measured noninvasively with near-infrared optical imaging," Psychophysiology 40, 548-560 (2003).
50. R. M. Birn et al., "fMRI in the presence of task-correlated breathing variations," Neuroimage 47, 1092-1104 (2009).

51. R. B. Buxton, E. C. Wong, and L. R. Frank, "Dynamics of blood flow and oxygenation changes during brain activation: the balloon model," Magn. Reson. Med. 39, 855-864 (1998).

Biographies for the authors are not available. 